\title{
ActiDote - A wireless sensor-based system for self-tracking activity levels among manual wheelchair users
}

\author{
Héctor F. Satizábal ${ }^{1}$, Alexandre Grillon ${ }^{1}$, Giacomo Picasso $^{3}$, Andres Upegui ${ }^{2}$, Grégoire Millet ${ }^{3}$, \\ Andres Perez-Uribe ${ }^{1, *}$ \\ ${ }^{1}$ Institute for Information and Communication Technologies, IICT. School of Engineering and Business of the \\ Canton of Vaud, HEIG-VD. University of Applied Sciences and Arts of Western Switzerland, HES-SO. \\ ${ }^{2}$ Institut d'ingénierie Informatique et des Télécommunications, InIT. Haute École du paysage, d'ingénierie et \\ d'architecture de Genève, HEPIA. University of Applied Sciences and Arts of Western Switzerland, HES-SO. \\ ${ }^{3}$ Institut des sciences du sport de l'Université de Lausanne, ISSUL. University of Lausanne, UNIL. Switzerland.
}

\section{Abstract}

ActiDote -activity as an antidote- is a system for manual wheelchair users that takes advantage of wireless sensors to recognize activities of various intensity levels in order to allow self-tracking of the physical activity. In this paper, we describe both the hardware setup and the software pipeline that enable our system to operate. Laboratory tests using multi-modal fusion and machine learning reveal promising results on classifying activity levels and assessing energy expenditure during wheelchair propulsion on ramps of different slopes and speeds. Our results indicate that it is possible to implement a system that uses the accelerometer of a smartphone as the only sensor in the wheelchair, i.e., by attaching it to the wheelchair frame. Additionally, the user might wear a smartwatch equipped with an accelerometer to enrich the system and enhance its performance.

Received on 15 September 2016; accepted on 10 June 2017; published on 18 July 2017

Keywords: Activity recognition, energy expenditure monitoring, self-tracking, wheelchair

Copyright (C) 2017 Héctor F. Satizábal et al., licensed to EAI. This is an open access article distributed under the terms of the Creative Commons Attribution license (http://creativecommons.org/licenses/by/3.0/), which permits unlimited use, distribution and reproduction in any medium so long as the original work is properly cited.

doi:10.4108/eai.18-7-2017.152896

\section{Introduction}

Physical inactivity has been identified as a major contributor to the exacerbation of physical illnesses [9]. The WHO identified it as the fourth leading risk factor of global mortality after high blood pressure, tobacco use and high blood glucose. Therefore, in recent years, many actions against inactivity have come to the fore [14]. For instance, diverse pedometer devices have been developed to help people reach various physical activity goals, like walking 30 minutes per day or completing 10'000 steps per day. Moreover, many smartphone applications attempt to help people self-track their physical activity and motivate them to continuously exercise. Unfortunately, an equivalent application wheelchair users is missing and there

${ }^{*}$ Corresponding author. Email: andres.perez-uribe@heig-vd.ch is a clear absence of motivational devices that can support the self-tracking of physical activity among people with motor disabilities. The few studies that have dealt with this issue concluded that current commercial physical activity measurement devices are not appropriate for wheelchair users [8], and to make things worse, those users very often adopt sedentary habits as a consequence of their disability. The result is that obesity rates for adults with disabilities are higher than for adults without disabilities [4].

In this paper we present ActiDote -activity as an antidote-: a system based on wearable and wheelchairattached sensors that wirelessly communicate with a smartphone to allow the tracking of the physical activity of people with motor disabilities using wheelchairs. This setup allowed us to develop machine learning models capable of estimating the energy 
expenditure (EE) of a wheelchair user adopting a multilevel modelling approach. The first level consists in building a data-driven model recognising the activities performed by the user (e.g., office work, locomotion, resting) and then estimate the EE using pre-computed activity-specific tables $[6,7,15]$. Moreover, given that the activity in which users spend more energy in most of the cases is related to locomotion, the second level focuses on this activity and builds a data-driven model of EE during locomotion using the measures of a portable metabolic cart (K5 from Cosmed, Rome, Italy) as ground-truth. We started by feeding our models with data from the whole set of available sensors (i.e., those attached to the wheelchair and those worn by the user), and then we found the best subset of sensors in terms of performance, availability, ease of installation and use. Moreover, we decided to keep the sensor's outputs in the international system of units (SI) to make the system easy to reproduce ${ }^{1}$. This choice allowed us to create a smartphone application that identifies the intensity of the physical activity being realized, integrating the machine learning algorithms that analyse the sensor data and provides a daily (and weekly) summary of activities as a feedback to the user.

This paper is organized as follows. Section 2 presents a summary of related works by other researchers. We highlight the differences and the common points with the proposed system. Section 3 briefly describes the hardware setup that was used during the experiments. Section 4 describes the ActiDote system and how the models of EE were built. The details of both levels of the system are given in Section 4.1 and Section 4.2 respectively. They include the details of the raw data and the features that were computed, as well as the details of the models used for assessing EE. Section 5 lists the results we obtained from the tests in a controlled environment. Section 5.1 and Section 5.2 show the performance of both levels of the system which were evaluated using two independent datasets. Moreover, Section 5.3 briefly presents the web application that was developed as a means to give a feedback to the users. Finally Section 6 concludes the manuscript by summarizing the current state of the project and giving some insights into further work that should improve the performance of the system.

\section{Related Work}

The increasing availability of wearable sensors embedded in smartphones, watches and physical activity trackers has opened the door to a wide number of applications, mainly in health and wellness improvement. Many devices and services permit to monitor

\footnotetext{
${ }^{1}$ Avoiding the use of the so called "activity counts" delivered by some commercial platforms.
}

physical activity, caloric intake, sleep quality, posture, and other factors involved in personal well-being (e.g., the so-called Quantified-Self movement ${ }^{2}$ ). Typically, commercial trackers collect data by means of sensors like GPS, accelerometers, gyroscopes, barometers, heart rate meters, thermometers, microphones, etc. As far as the physical activity monitoring is concerned, recent research and development has allowed to leverage the power of accelerometers for building systems capable of estimating energy expenditure (EE). Staudenmayer et al. [17] used a single axis accelerometer and datadriven models based on artificial neural networks to estimate physical activity metabolic equivalents (MET) and to classify activity into low-level activities, locomotion, vigorous sports, and household activities. Vyas et al. [19] show how the BodyMedia FIT (now Jawbone) armband system was able to track EE by using machine learning methods that fused the information of several sensors (e.g., skin temperature, galvanic skin response, heat flux, acceleration). Ruch et al. [15] compared two machine learning approaches for estimating EE in children. They compared the performance of artificial neural networks vs. building a decision tree to classify the activities and then using tables to estimate energy expenditure. Even if both approaches provided good estimations, they concluded that the use of activitytype-specific information for subsequent EE prediction might be a promising approach for future studies. Some of these approaches to estimate energy expenditure have achieved mass market penetration (e.g., Jawbone, Fitbit, Nike+, Polar Loop, Garmin vivofit) and are available to the general public nowadays. Pande et al. [11] compared the performance of some of the commercial devices (i.e., Fitbit and Nike+ Fuel Band) with an online estimation of EE made from the information collected through the sensors in a smartphone (i.e., accelerometer and barometer) and a machine learning model based on artificial neural networks.

Unfortunately, it has been found that these general public devices do not provide accurate estimates of the energy expenditure of people using manual wheelchairs, in particular, during wheelchair propulsion $[3,8]$. Even the SenseWear Armband (SWA), which has been validated as a means to estimate energy expenditure in overweight children, in patients with cancer, and healthy children, has provided inaccurate measures among the disabled population [18].

Several researchers have addressed the problem of assessing energy expenditure of manual wheelchair users providing alternative solutions to the general public devices. Hiremath and Ding [7] tested an accelerometer-based activity monitor in the task of estimating EE. They fitted linear regression models

\footnotetext{
${ }^{2}$ http://quantifiedself.com
} 
by using information from two sensor locations i.e., waist and upper arm, and concluded that the latter gave more relevant information when predicting energy expenditure. Gendle et al. [6] were able to discriminate whether a group of users performed low or moderate effort. They obtained these encouraging results by using information from an accelerometer mounted on the frame of the wheelchairs. Nightingale et al. [10] tested the ActiGraph GT3X+ and found that this device was a reliable tool for determining mechanical movements within the physiological range of human movement. They built linear regression models using data from three body locations: right wrist, upper arm and waist. Moreover, they concluded that the model built from the acceleration measured at the wrist explains more of the variance and results in the lowest random error when predicting physical activity energy expenditure in manual wheelchair users. Garcia-Masso et al. [5] used the Actigraph GT3X and extracted thirty-six features from the acceleration signals. They built linear regression models to estimate $\mathrm{EE}$ and concluded that the sensor placed on the non-dominant wrist gave the best results.

Herein, we report a solution that takes advantage of some features that have proven useful in previous works, i.e., using an inertial sensor on the wrist (which can be easily worn), classifying activities into different categories, using pre-computed tables of physical activity intensity and modelling EE by using simple regression models. On top of that, our work improves the experimental setup by considering the case of selfpushing the wheelchair through negative and positive slopes. Last but not least, the ActiDote system does not use the so-called "activity counts" delivered by some devices (e.g., Actigraph GT3X) but uses acceleration and speed data in raw SI units (i.e., $\left[\frac{\mathrm{m}}{\mathrm{s}^{2}}\right]$ and $\left[\frac{\mathrm{rad}}{\mathrm{s}}\right]$ ) to make it easier to export our findings to general public applications.

\section{Hardware Setup}

We endowed an ordinary manual wheelchair with a set of wireless sensors allowing to capture relevant information to assess the energy expenditure of a person. We based the selection of sensors on two hypotheses:

- The type of activity can be estimated using information captured with inertial sensors placed on the wheelchair and on the user body

- It is impossible to accurately infer activity intensity from inertial motion sensors only

The first hypothesis comes from previous experiences done by other researchers. The second hypothesis comes from some observations e.g., displacing a wheelchair on

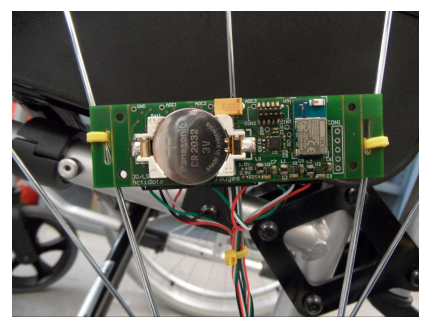

(a) Wireless sensor board



(c) Wheelchair, smartwatch and the modified wheel

Figure 1. Hardware setup

a regular surface will require much less energy than on a sandy surface, and it can be more physically intensive to stay static on an uphill than to move on a downslope. In both cases inertial sensors capture very similar data.

We have thus equipped an ordinary wheelchair with a set of Bluetooth Low Energy (BLE) sensors in order to build a complete physical activity monitoring system. We designed and build two sensing boards containing a gyrometer, a accelerometer and some additional analog inputs. One of these boards was fixed on the wheelchair frame to measure the accelerations of the wheelchair (and hence its tilt). The other board was fixed on the wheel to measure the speed of the wheel and hence the linear speed of the wheelchair (see Figure 1a).

Moreover, we modified one of the wheels in order to assess the forces applied to it. We replaced the hand rim separators by three load cells which measure the tangential force applied to the wheel (by measuring the mechanical deformation between the hand rim and the wheel). Figure $1 \mathrm{~b}$ depicts how each load cell has been coupled to the wheel. The strain gauges on each load cell are connected to form a Wheatstone bridge topology. The two output signals from the bridges are then amplified, compared and filtered. Finally, the resulting signal is read by the analog inputs in the sensing board. Figure 1c shows the complete hardware setup.

Both sensing boards are equipped with a Bluetooth Low Energy (BLE) module. The module is the 
BLE113 developed by Bluegiga. It integrates a calibrated antenna at $2.4 \mathrm{GHz}$ and a low consumption microcontroller. The main advantage of this all-in-one module is the reduction of the development time on the hardware design and the full implementation of the Bluetooth stack usable with a simple script language named BGScript.

Finally, a commercial smartwatch (i.e., the Moto 360) is fixed on the user's wrist. It is also equipped with a gyrometer and an accelerometer and is used to detect arm motions in order to improve the model with the detection of other gestures (e.g., auto-propulsing the wheelchair, playing ping-pong or lifting weights). Moreover, this smartwatch is also equipped with a photoplethysmogram sensor used to monitor the user's heart rate (HR). HR measurements should help to enhance the model by directly using physiological data for estimating specific users caloric expenditure needs and capacity.

All the data from custom sensors and the commercial smartwatch are transferred through BLE to an Android handheld device carried by the wheelchair user in order to further perform data analysis.

\section{Experimental Setup}

The amount of energy spent by a person during a given activity can be assessed using a mechanistic approach if the right physiological variables are measured. However, these measurements are not easy to perform due to different practical constraints (e.g., sensors are expensive and not portable). Data-driven approaches offer an alternative to the mechanistic analyses which have been traditionally used for modelling complex metabolic phenomena. Data-driven models try to discover the relationships between the variables involved in the analysis from the data, while in mechanistic approaches these relationships are based on prior theoretical knowledge about the phenomenon. Indeed, in a data-driven approach, the variables used do not necessarily have a physiological meaning, and in most of the cases they are mere surrogates to a quantity that is difficult to measure. One of the main strengths of a data-driven approach is its robustness. The fact of using the acquired data to build the model provides a better tolerance to sensor imprecision given its nondependence on sensor calibration or precise sensor positioning and orientation. While mechanistic analysis relies on the understanding of a physical phenomena that requires well defined inputs for obtaining an accurate solution, data-driven approaches accept to do not understand the phenomena, making it less dependent on the accuracy of the inputs. Our approach is thus to acquire as much pertinent sensor data as possible for further identification of the required inputs to build the model.

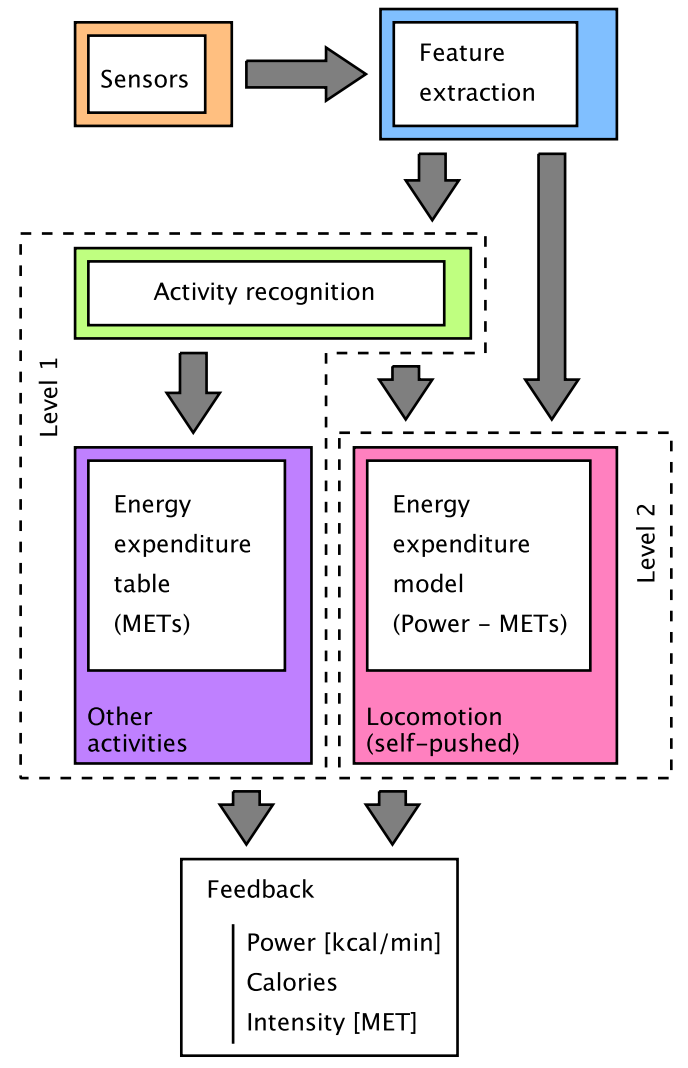

Figure 2. Block diagram of the ActiDote setup

The ActiDote system implements two levels of datadriven modelling. The first level is a classification model that must recognize which type of activity is being done by the wheelchair user (green block in Figure 2). Hence, it is possible to use pre-defined tables of physical activity [1] to assess the actual energy expenditure of the user in near real-time. The second level is a regression model that is triggered only when locomotion takes place (see pink block in Figure 2), and must estimate the energy expenditure of the user from the information provided by some sensors installed on the wheelchair and/or worn by the person.

The outputs of both layers (pre-computed tables and models) are transformed into metabolic equivalents (MET) to give a feedback of physical activity intensity to the user.

The ActiDote system gathers data from the inertial sensors mounted on the wheelchair and worn by the user, and from the force sensors mounted on one of the wheels of the wheelchair. For model calibration purposes, it also monitors the gas exchanges of the user by using the $\mathrm{K} 5$ portable metabolic cart ${ }^{3}$. Table 1 shows a list of the most relevant variables that were

\footnotetext{
${ }^{3}$ Cosmed, Rome, Italy
} 
Table 1. List of the most important available variables. The strain gauges were mounted on the wheel. The Chair sensor was mounted on the frame of the wheelchair

\begin{tabular}{llll}
\hline Variable name & N. of axes & Units & Source \\
\hline Wrist acceleration & 3 & $\mathrm{~m} / \mathrm{s}^{2}$ & Watch \\
Wrist angular speed & 3 & $\mathrm{rad} / \mathrm{s}$ & Watch \\
Wheel acceleration & 3 & $\mathrm{~m} / \mathrm{s}^{2}$ & Wheel sensor \\
Wheel angular speed & 3 & $\mathrm{rad} / \mathrm{s}$ & Wheel sensor \\
Wheel force & 3 & $\mathrm{Nw}$ & Strain gauges \\
Chair acceleration & 3 & $\mathrm{~m} / \mathrm{s}^{2}$ & Chair sensor \\
Chair angular speed & 3 & $\mathrm{rad} / \mathrm{s}$ & Chair sensor \\
Heart rate & 1 & $\mathrm{bpm}$ & $\mathrm{K} 5$ \\
VO2 & 1 & $\mathrm{ml} / \mathrm{min}$ & $\mathrm{K} 5$ \\
VCO2 & 1 & $\mathrm{ml} / \mathrm{min}$ & $\mathrm{K} 5$ \\
VE & 1 & - & $\mathrm{K} 5$ \\
\hline
\end{tabular}

collected during the experiments. After some initial tests, we determined that the heart rate measures given by the smartwatch were too noisy and divergent to be used. Therefore, we decided to only keep the heart rate measures given by the $\mathrm{K} 5$.

\subsection{Activity Recognition}

In order to recognize different activities, we started by determining the activities that should be detected and learnt. We included activities such as: resting, moving (e.g., at different speeds, in different ground surfaces, given different slopes, self-pushed, pushed by a caretaker), desk-work-like (e.g., desk work, working on a computer, eating, being on the phone) or even replacements (e.g., in the chair, from the wheelchair to the toilet). These activities were grouped in several classes of intensity ranging from sleeping (or None) to vigorous. For the machine learning to take place, the first step consisted in collecting data while performing the activities we mentioned above. This operation was carried out by an Android handheld device. The data captures followed a protocol that encompassed five activities: resting, desk work, self-pushing at medium speed on a standard flat indoor floor, self-pushing on an ascending slope, and external pushing by a caretaker. Each activity defined in the list was associated to an intensity level: none, light, moderate and vigorous. The protocol required no predefined order for doing the activities, but defined that each activity had to be performed for five minutes straight. This was a precaution to make sure that the model had enough examples of each activity and was therefore able to learn all of them correctly. This protocol was obviously only designed for laboratory purposes and was used to train a model to recognize such activities. The long term goal was to be able to classify captures free from any protocol, such as captures that any wheelchair user would do in a normal day. To ensure the activity recognition model performed well, we chose to train it on a specific data capture, and then to test it on another data capture and the other way around. This way, we could be sure that our predictive model generalized well on the activities themselves and not on setup dependent variables. Furthermore, we grouped the obtained data following an early fusion pattern and downsampled the Moto 360 accelerometer data by decimation from $50 \mathrm{~Hz}$ to $15.635 \mathrm{~Hz}$. Therefore, at the end of the data collection step, we had two fully labelled datasets of roughly 25 minutes with 5 minutes by activity and a $15.635 \mathrm{~Hz}$ sampling rate.

Feature extraction. We rolled two windows of respectively 5000 and $1000 \mathrm{~ms}$ over the data gathered from the wheelchair sensors (not the K5) and we computed the median, standard deviation and energy of the signal of the windows leading to each sample (included). We were therefore left with two datasets of respectively 22471 samples (24 minutes) and 23439 samples (25 minutes).

Additionally, we prepared alternative datasets by hand crafting and hand-picking some of the features that we know are good discriminants of the activity classes we have. These features were namely: the median chair acceleration in the $\mathrm{x}$-axis over the last $5000 \mathrm{~ms}$, the magnitude of the standard deviation of the chair three-axis accelerometer values over the last $1000 \mathrm{~ms}$, the median wrist acceleration in the $\mathrm{x}$-axis over the last $5000 \mathrm{~ms}$, the magnitude of the standard deviation of the wrist three-axis accelerometer values over the last $5000 \mathrm{~ms}$.

Modelling tool. As a lot of the extracted features are redundant and correlated, the best choice for an offthe-shelf model was random forest (RF). This is a model introduced by Breiman in 2001 [2] that bags (short for bootstrap aggregates) classification and regression trees (CART) and randomly samples a subset of the features at each split. In doing so, the model averages many noisy but approximately unbiased models, and hence reduces the variance. For classification, a committee of trees casts a vote for the predicted class. As trees are invariant under linear modification of input, this also speeds up the activity recognition chain since no further preprocessing is required. The splitting criteria was the entropy and the number of estimators in the RF model was 1000 .

\subsection{Energy Expenditure during Locomotion}

In order to build data driven models of energy expenditure, we collected data following the experimental protocol shown in Table 2. 
Table 2. Experimental protocol used for capturing data on the treadmill. Each checked cell represents a test composed of five minutes of recovery time plus five minutes of activity. Unchecked cells represent tests that were dangerous or too difficult

\begin{tabular}{c|cccccc} 
Speed & $-4 \%$ & $-2 \%$ & $0 \%$ & $2 \%$ & $4 \%$ & $6 \%$ \\
\hline $2\left[\frac{\mathrm{km}}{\mathrm{h}}\right]$ & $\checkmark$ & $\checkmark$ & $\checkmark$ & $\checkmark$ & $\checkmark$ & $\checkmark$ \\
$4\left[\frac{\mathrm{km}}{\mathrm{h}}\right]$ & $\checkmark$ & $\checkmark$ & $\checkmark$ & $\checkmark$ & $\checkmark$ & \\
$6\left[\frac{\mathrm{km}}{\mathrm{h}}\right]$ & & & $\checkmark$ & & &
\end{tabular}

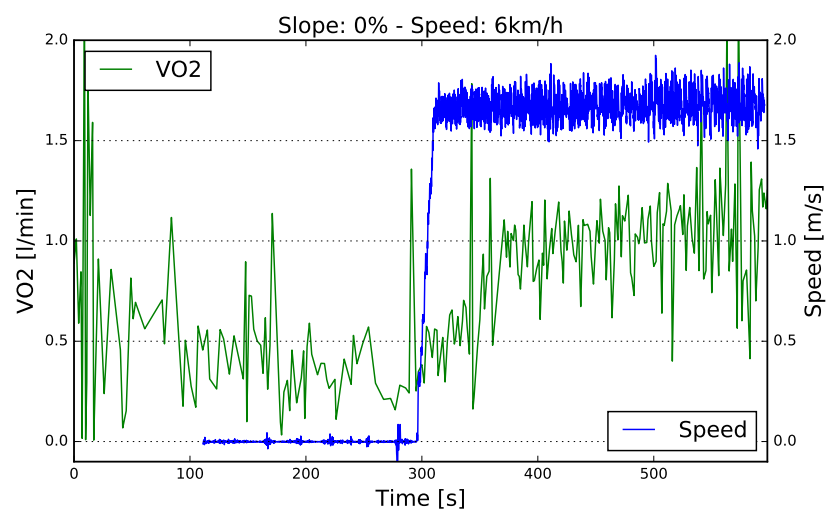

Figure 3. Example of one of the tests in the protocol

We used a large treadmill ${ }^{4}$ to set the speed and inclination of the wheelchair, and a K5 portable metabolic cart $^{5}$ to quantify the actual energy expenditure of the user. Calibration procedures were performed before each session according to the manufacturer's instructions. Each checked cell in Table 2 represents a test of 10 minutes: 5 minutes of rest time and 5 minutes of activity. Unchecked cells represent tests that were dangerous or too difficult. The treadmill did not move during the first five minutes. During this time the user rests in the wheelchair and should remain relaxed in order to recover from the previous effort. Once the five minutes of recovering have passed, the treadmill starts moving at the desired speed (blue line in Figure 3) and hence the user starts to consume oxygen (green line). As it can be seen in Figure 3, the variables measured by the metabolic cart needs some time to reach its final and stable value. Once the stable value is reached, we can speculate that the exercise is purely aerobic, and that the respiratory exchange ratio (RER) is stable. Therefore, we only consider as valid data the last three minutes of each part of the test.

\footnotetext{
${ }^{4}$ Saturn HP Cosmos, Traunstein, Germany

${ }^{5}$ Cosmed, Rome, Italy
}

Table 3. Description of the three subjects who participated in the treadmill experiments. Age is expressed in years, weight in kilograms and height in centimetres

\begin{tabular}{lrrrrr}
\hline & Age & Weight & Height & Gender & N. tests \\
\hline Subject 1 & 36 & 73 & 175 & $\mathrm{M}$ & 2 \\
Subject 2 & 24 & 87 & 184 & $\mathrm{M}$ & 1 \\
Subject 3 & 26 & 53 & 158 & $\mathrm{~F}$ & 1 \\
\hline
\end{tabular}

Table 3 shows a characterization of the three subjects who participate in the experiments.

Feature extraction. Table 1 shows a list of some of the available variables. The variables provided by the sensors installed on the wheelchair were sampled at a fixed frequency of $15.635 \mathrm{~Hz}$ (i.e., sampling period of $0.064 \mathrm{~s})$. On the other hand, the sampling frequency of the variables measured by the metabolic cart depends on the breathing frequency of the subject. Thus, during a pre-processing step we transformed the data gathered by the metabolic cart and put the whole set of variables to the same frequency.

Moreover, we extracted time windows of 30 seconds of length from the data within the last 3 minutes of each part of the test (i.e., recovery and activity) and we computed the average and the standard deviation of the data in these windows.

The variable we want to predict is the energy expenditure (EE) of the person on the wheelchair. To determine the EE during exercise we used the measure of oxygen metabolised by the person (i.e., VO2) given by the portable metabolic cart. We transformed the VO2 into kilo-calories per minute $[\mathrm{kcal} / \mathrm{min}]$ as it is shown in equation 1 .

$$
\begin{aligned}
1 M E T & :=\frac{1 k c a l}{k g \cdot h}:=\frac{3.5 m l O_{2}}{k g \cdot m i n} \\
E E\left[\frac{k c a l}{h}\right] & =V O 2\left[\frac{m l}{m i n}\right] / 3.5 \\
E E\left[\frac{k c a l}{m i n}\right] & =V O 2\left[\frac{m l}{m i n}\right] / 210 \\
M E T s & =\frac{60 \cdot E E\left[\frac{k c a l}{m i n}\right]}{b o d y \_m a s s[k g]}
\end{aligned}
$$

We used the value of EE in kilo-calories per minute as target of the models we want to build. We preferred to measure the $\mathrm{EE}$ in kilo-calories per minute since these units are better suited for the usage of daily life ${ }^{6}$. However, it is possible to express EE (power) and energy in the international system of units (Watts [W] and

\footnotetext{
${ }^{6}$ People are already accustomed to measure energy in calories when assessing metabolic rate and metabolic intake.
} 
Joules [J]) if the ActiDote system is used as a means to assess energy expenditure in studies of physical disability. Equation 3 shows how to transform the value of $\mathrm{VO} 2$ into power in Watts.

$$
\begin{aligned}
1 \mathrm{cal} & :=4.18 \mathrm{~J} \\
1 \frac{k c a l}{\mathrm{~s}} & =\frac{4.18[\mathrm{~J}] \cdot 1000}{1[\mathrm{~s}]}=4180\left[\frac{\mathrm{J}}{\mathrm{s}}\right]=4180[\mathrm{~W}] \\
\operatorname{EE}\left[\frac{k c a l}{\mathrm{~s}}\right] & =\operatorname{VO} 2\left[\frac{\mathrm{ml}}{\min }\right] / 12600 \\
\text { Power }[\mathrm{W}] & \approx \operatorname{VO} 2\left[\frac{\mathrm{ml}}{\min }\right] / 3
\end{aligned}
$$

Moreover, equation 2 shows how to compute metabolic equivalents (MET) from the results given by the model.

Modelling tool. Figure 4 shows the correlation between the EE and other available variables (i.e., features from sensor data). Only correlations higher than 0.52 (correlation between EE and time) are shown.

As it can be seen in Figure 4, there are a lot of variables which are highly correlated with the EE of the subject. These high correlations indicate that the relationship between EE and the available variables are mostly linear, or at least, that they can be explained by a linear model. Following this intuition, we decided to fit some linear regression models to predict the EE of the person as a function of some subsets of available variables.

\section{Results}

This section summarizes the results of the two modelling layers of the ActiDote system.

\subsection{Activity Recognition}

Given that we are mainly interested in having a global feedback of the activities done during the day, we filtered the output of the classifier using a modal filter of 1 minute to reject errors due to fast variations in the classification of activities.

To assess the performance of the models we performed a leave-one-test-out validation scheme in which we used one dataset for building the model and the other for validating it. We repeated this crossvalidation 10 times to ensure that the results are not just found by chance. Finally, the classification performance was measured with the F1-Score (weighted averaged over all the tests and all the classes).

We employed the scikit-learn [13] package of Python to train and validate the random forest classification models.
Table 4. F1-scores on training and validation datasets for both training orders

\begin{tabular}{cccc}
\hline Training Set & Prediction Set & Train & Validation \\
\hline Dataset 1 & Dataset 2 & $0.99 \pm 0.00$ & $0.77 \pm 0.03$ \\
Dataset 2 & Dataset 1 & $0.99 \pm 0.00$ & $0.99 \pm 0.00$ \\
\hline
\end{tabular}
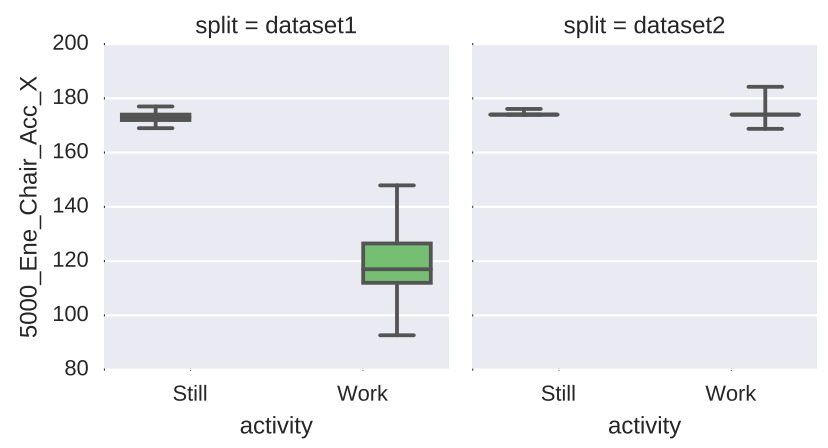

Figure 5. Example of a feature leading to a wrong classification of activities Still and Work. The first dataset on the left side, the second one to the right

Using all features. Table 4 shows the performance of the classification of activities when all the features are used as inputs to the models. Each row in the table represents a different order for training and validation, each column contains results on the training set (by outof-bag score) and on the validation set.

As we see in Table 4, both ways of training and validation are not equivalent. Indeed, the score on the validation set when trained on the first dataset drops dramatically compared to the same score when trained on the second one. This indicates a disparity between the two captures which has important effects on the final performance given that the datasets are small. Some features, like the energy of the chair accelerometer signal in the $\mathrm{x}$-axis over the last $5000 \mathrm{~ms}$ which is shown in Figure 5, can be discriminant on one of the datasets but not in the other one.

Such problems should disappear if the models are built using more data, capturing a more general view of the process being modelled. Another way of overcoming that issue would be to manually select features that we know from expert knowledge are relevant for characterizing the activities we defined.

Manually selected features. In this section we show the results we obtained when using the features described in Section 4.1. The performance of the models built from those features are presented in Table 5. Moreover, Figure $6 a$ depicts the confusion matrices of ground truth against predictions with the first dataset used for training and the second dataset used for validating. 




Figure 4. Highest correlation values between each available variable and the $E E$

Table 5. F1-scores on training and validation datasets with manually selected features

\begin{tabular}{cccc}
\hline Training Set & Validation Set & Train & Validation \\
\hline Dataset 1 & Dataset 2 & $0.99 \pm 0.00$ & $0.94 \pm 0.02$ \\
Dataset 2 & Dataset 1 & $0.99 \pm 0.00$ & $0.98 \pm 0.00$ \\
\hline
\end{tabular}

Figure $6 \mathrm{~b}$, on the other hand, shows the same matrices but with reverse training-validation order.

\subsection{Energy Expenditure during Locomotion}

In order to chose the best combination of inputs and the best model, we perform a leave-one-testout validation scheme. We fit four models, each time keeping apart the data of one of the test to assess the validation performance. In each case we computed the coefficient of determination (R-squared) between the predicted and the target values. We employed the statsmodels [16] package of Python to build and validate the linear regression models. Table 6 shows the results we obtained.

As it can be seen in Table 6, the best results are obtained when using features only from the wheelchair and the wrist $\left(R^{2}=0.859\right)$. The worse results were obtained when using variables from the wheel only, especially in the case of S3. It seems that the distribution of the features computed from the wheel data are very different when subject S3 pushed the wheelchair. Features computed from wheel data even decrease the performance in they are used with features computed from chair data $\left(R^{2}=0.809\right.$ to $R^{2}=0.754$ and $R^{2}=0.809$ to $\left.R^{2}=0.745\right)$. Besides, placing sensors on the wheel is not practical if one

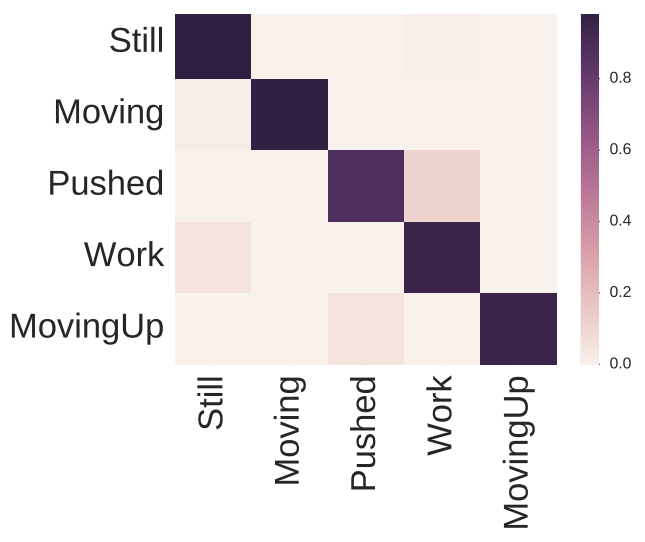

(a) Using the first dataset for training and the second dataset for validating

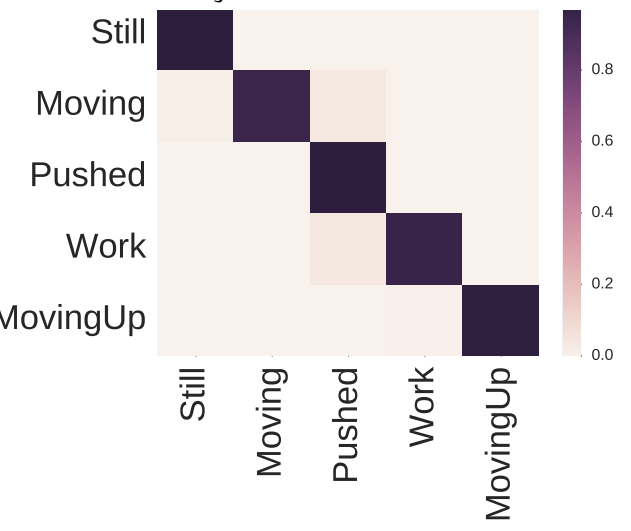

(b) Using the second dataset for training and the first dataset for validating

Figure 6. Confusion matrices of the models built with the manually selected features 
Table 6. Performance $\left(R^{2}\right)$ of the models built with different combinations of inputs

\begin{tabular}{lrrrrr}
\hline Description & S1 R1 & S1 R2 & S2 & S3 & Mean \\
\hline all features (af) & 0.889 & 0.913 & 0.842 & 0.774 & 0.855 \\
af - no load cells & 0.875 & 0.891 & 0.764 & 0.829 & 0.840 \\
chair features & 0.887 & 0.866 & 0.674 & 0.809 & 0.809 \\
wheel features (wf) & 0.707 & 0.723 & 0.681 & 0.051 & 0.540 \\
wf - no load cells & 0.446 & 0.608 & 0.579 & 0.016 & 0.412 \\
wrist features & 0.857 & 0.889 & 0.822 & 0.791 & 0.840 \\
chair and wheel (cw) & 0.863 & 0.881 & 0.767 & 0.505 & 0.754 \\
cw - no load cells & 0.818 & 0.795 & 0.650 & 0.716 & 0.745 \\
wheel and wrists (ww) & 0.884 & 0.883 & 0.852 & 0.751 & 0.843 \\
ww - no load cells & 0.874 & 0.871 & 0.855 & 0.808 & 0.852 \\
chair and wrist & 0.899 & 0.925 & 0.773 & 0.838 & 0.859 \\
\hline
\end{tabular}

thinks about building an end-user system. On the other hand, features computed from wrist data seem to increase the performance in every case i.e., chair and wrist is better than chair only $\left(R^{2}=0.859\right.$ vs. $R^{2}=$ $0.809)$, wheel and wrist is better than wheel only $\left(R^{2}=\right.$ 0.843 vs. $R^{2}=0.540$ and $R^{2}=0.852$ vs. $R^{2}=0.412$ ). Indeed, models built from only wrist data perform quite well $\left(R^{2}=0.840\right)$. As far as the chair features are concerned, they are not the best nor the worse in the ranking. Chair features have a good performance $\left(R^{2}=\right.$ $0.809)$ compared to wheel features, with the additional advantage of being easy to obtain given that installing sensors on the wheelchair frame is easier than installing sensors on the wheel.

Another interesting conclusion to draw from this analysis is that whether the model uses or not the force sensors (load cells), it does not seem to greatly influence the performance of the resulting model. In one of the cases i.e., wheel and wrist, it is even better not to use the features coming from the load cells. The hypothesis that the force would be a good predictor of the energy when the wheelchair goes up through a slope does not hold for the experiments we performed. However, more experiments need to be done in order to reject this hypothesis especially in the case of static efforts or diverse ground surfaces.

It is worth to notice that subject S1 participated twice in the tests and hence two of the datasets were collected by the same person. Therefore, during repetitions S1 R1 and S1 R2 of the cross-validation tests, one of the datasets in the training data was generated by the same user generating the validation data ${ }^{7}$. This explains why the performance of the models when $\mathrm{S} 1$ is used to assess the performance are always a little bit higher.

\footnotetext{
${ }_{7}^{7}$ The data are not identical, they were collected during two different sessions, two different days.
}

Towards a Mobile Application. Using the results shown in Section 5.2 as guidance, we performed a selection of the most interesting features to build a real world implementation. We considered two use cases: (1) the user has a smartphone that can be attached somewhere to the wheelchair frame and (2) the user additionally has a smartwatch connected to her/his smartphone. Even if it would increase the performance of the system, we did not consider the case of installing sensors on the wheel since it would make the installation of the system more complicated and thus, less users would have access to the application.

We applied a backward elimination scheme of feature selection to the two aforementioned scenarios i.e, chair features, and chair and wrist features. Figure 7 shows the performance of the resulting models.

In the case of using features from the wheelchair frame only, we found that it is possible to build an accurate $\left(R^{2}=0.81\right)$ linear regression model using the standard deviation of a single axis of the accelerometer i.e., the axis of movement $(X)$. Those results are very encouraging because it means that a light smartphone application could deliver good approximations of energy expenditure if the smartphone is attached to the wheelchair frame. The linear model using a single feature from the wheelchair frame is shown in Equation 4 , where $F_{1}$ is the standard deviation of the $X$ axis of the accelerometer attached to the wheelchair frame.

$$
E E=4.263 \cdot F_{1}+1.252
$$

On the other hand, using features from the wheelchair frame and from the wrist it is possible to build a slightly better model $\left(R^{2}=0.82\right)$ which employs two additional features: the average and the standard deviation of the acceleration of the wrist (magnitude). The linear model using a single feature from the wheelchair frame and two features from the wrist is shown in Equation 5, where $F_{1}$ is the standard deviation of the $X$ axis of the accelerometer attached to the wheelchair frame, $F_{2}$ is the average of the magnitude of the acceleration measured at the wrist and $F_{3}$ is the standard deviation of the magnitude of the acceleration measured at the wrist.

$$
E E=2.964 \cdot F_{1}-0.577 \cdot F_{2}+0.564 \cdot F_{3}+6.842
$$

Both solutions need to be further tested in order to evaluate their robustness and to know how they behave in different real world situations (e.g., locomotion over different ground surfaces).

\subsection{Feedback Visualization}

A graphical summary of the amount of time spent on activities of different levels of intensity can be exploited 


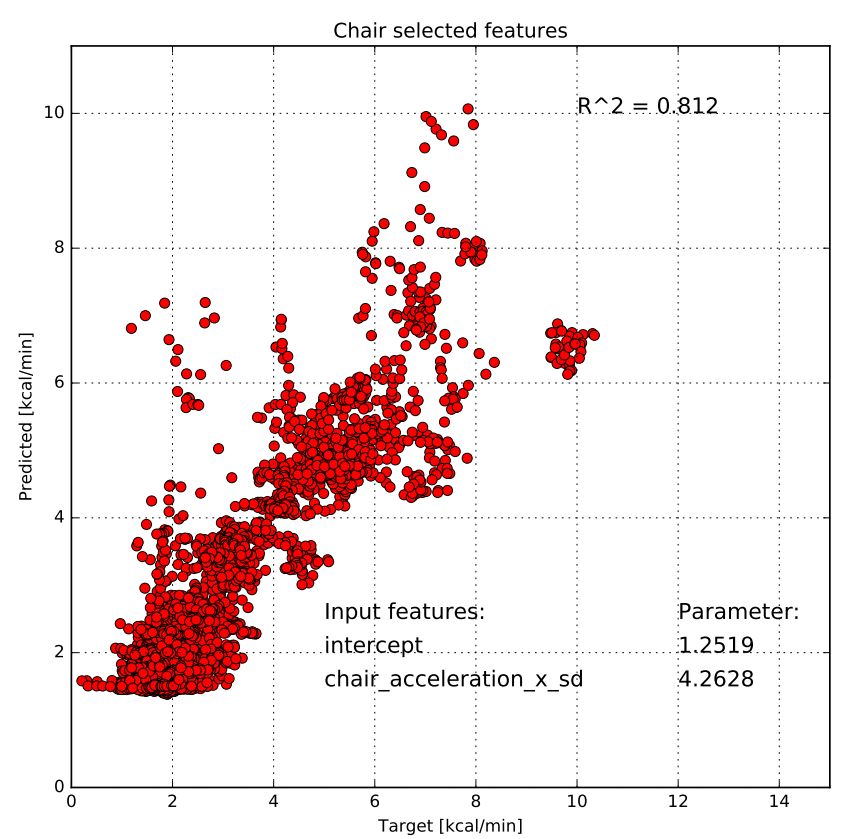

(a) Chair frame subset

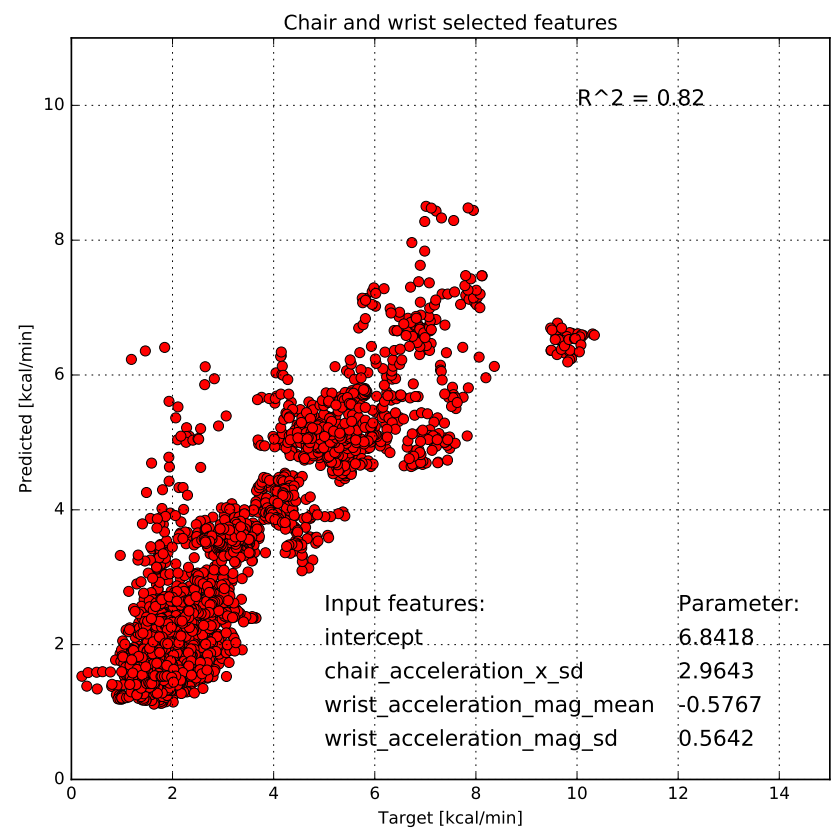

(b) Chair frame and wrist subset

Figure 7. Results of the models created after undergoing the feature selection process. The $R^{2}$ values shown were computed using cross-validation

in a straightforward way to motivate more regular physical exercise. We developed a web interface that shows both the current day summary and a history of the previous week. The interface in its current state is depicted in Figure 8.
The application gives estimations of the amount of calories burnt by using two approaches. On the one hand it employs the classification made by the first model (i.e., random forest) for indexing a table of pre-computed values of MET [1]. On the other hand, and only if the wheelchair user is pushing her/his wheelchair (i.e., locomotion), the application employs the second model (i.e., linear regression) to compute an estimation of the energy expenditure in kilo-calories per minute. This estimation is more specific and thus precise since it considers more variables compared to a pre-computed table which does not take into account variables like the acceleration or the speed. Kilo-calories per minute are then transformed to MET to have a measure of activity intensity.

Moreover, the statistics shown by the application are related to the following intensity levels: Sleeping (or None), Light, Moderate, and Vigorous. These levels were computed by using the model proposed by Pate et al. [12] for classifying the MET intensity of physical activities as follows: light, $<3$ METs; moderate, 3-6 METs; vigorous, $>6$ METs.

\section{Conclusions}

The ActiDote system aims at providing a solution to the problem of assessing energy expenditure in the case of wheelchair users. By using a data-driven approach it is able to classify activities belonging to different levels, and to use this classification to convey a meaningful value of energy expenditure. For the specific case of locomotion, ActiDote is able to compute a more precise estimation of the energy expenditure by indirectly taking into account more information e.g., the speed of the wheelchair and the inclination of the terrain., by means of sensors already present in a mobile device.

Compared to the results presented in other state-ofthe-art works $[5-7,10]$, the ActiDote system has been additionally tested considering wheelchair propulsion on ramps of different slopes. The outcome is a model that yields meaningful estimations in a larger set of conditions. However, the results we show in this article come from tests made at the laboratory, and therefore more tests are needed before claiming a correct estimation in a real-world environment. We need to further test the complete system particularly in e.g., different ground surfaces, people with a wider range of ages.

The final setup of ActiDote does not employ the load cells installed on the wheel of the wheelchair. This is positive since it makes possible to implement the system using the accelerometer in a smartphone as the only sensor in the wheelchair (by attaching the smartphone to the wheelchair frame). Additionally, the user should wear a smartwatch to enrich the system and ameliorate its behaviour. On the other hand, without 

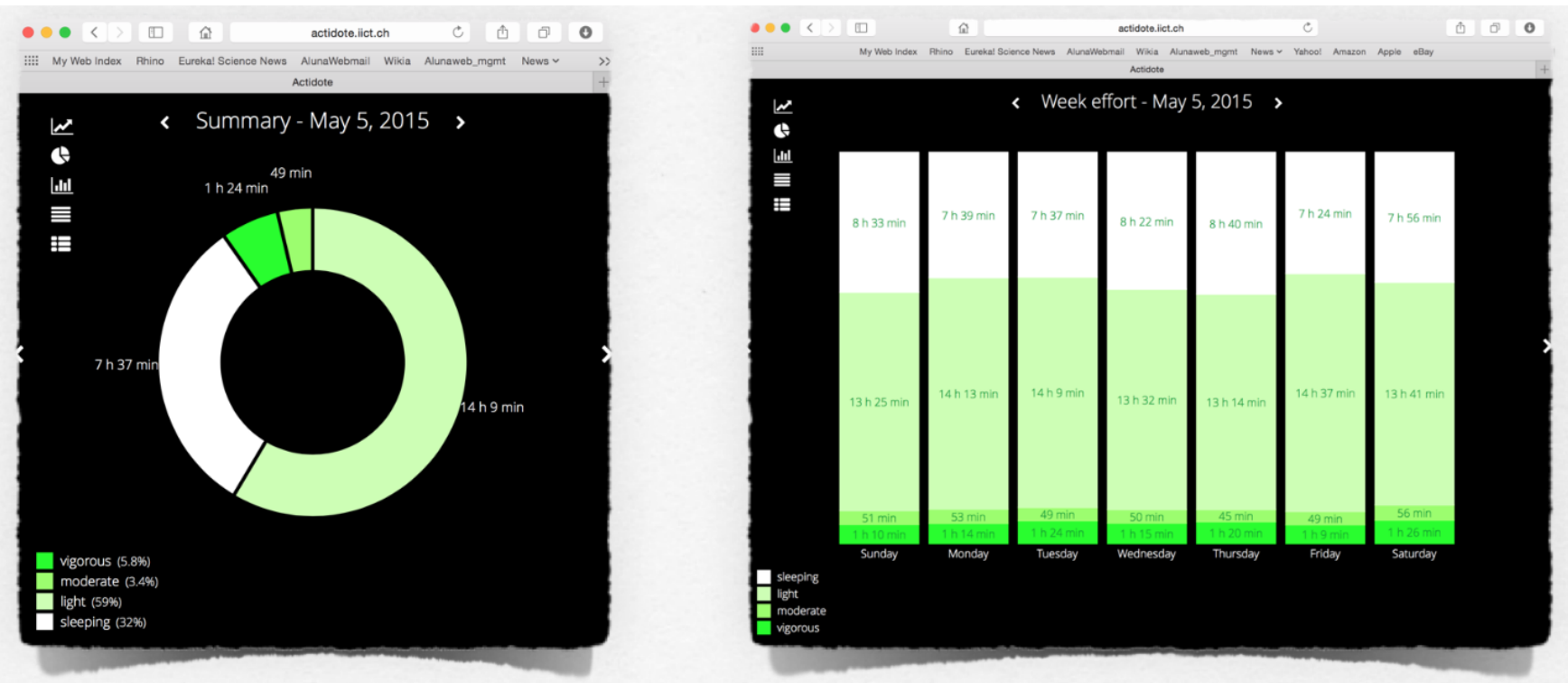

Figure 8. The web interface for the end user feedback

the force sensors it would be impossible to differentiate between different ground surfaces, and to correctly assess the EE during static efforts. Further tests need to be done in order to assess to what extent the load cells are not necessary to estimate with little error the EE of the user.

Using two levels of modelling i.e., first performing activity recognition to make a coarse estimation of $\mathrm{EE}$ and then improving the estimation for the most significant class using specific models, also have some limitations. This approach implies that the designer of the system has to know the relevant activities beforehand in order to create a classifier that is able to perform in the specific context of each user. In most of the cases a set of general activities should work i.e., rest, desk-work, locomotion; but it is impossible to be sure that these activities will match the preferences of every user of the system.

We still need to embed the activity recognition algorithms into the current version of the smartphone application and detect whether the user pushes the wheelchair her/himself or if it is being pushed by a care-taker. In our current work, we considered selfpushing and being pushed as two different activities and managed to discriminate between them.

Last but not least, to evaluate the acceptability of the final application, we need to run the system on data gathered from real motor disabled people using our wheelchair.

Acknowledgements. This project has been supported by the HES-SO grant IA-INTERDISC14-01. We would like to thank all the members of the ActiDote consortium for fruitful discussions and collaboration and Eric Henchoz for developing the web-based visualization interface. We also would like to thank David Da Silva, Laurent Gantel and Clément Rastoll for the development of the sensors.

\section{References}

[1] Barbara E Ainsworth, William L Haskell, Stephen D Herrmann, Nathanael Meckes, David R Bassett, Catrine Tudor-Locke, Jennifer L Greer, Jesse Vezina, Melicia C Whitt-Glover, and Arthur S Leon. 2011 compendium of physical activities: a second update of codes and met values. Medicine and science in sports and exercise, 43(8):1575-1581, August 2011.

[2] Leo Breiman. Random forests. Machine Learning, 45(1):5-32, 2001.

[3] Scott Alexander Conger. Physical Activity Assessment in Wheelchair Users. PhD thesis, University of Tennessee, 2011.

[4] Weil E, Wachterman M, McCarthy EP, and et al. Obesity among adults with disabling conditions. JAMA, 288(10):1265-1268, 2002.

[5] X García-Massó, P Serra-Añó, L M García-Raffi, E A Sánchez-Pérez, J López-Pascual, and L M Gonzalez. Validation of the use of actigraph GT3X accelerometers to estimate energy expenditure in full time manual wheelchair users with spinal cord injury. Spinal Cord, 51(12):898 - 903, 2013.

[6] SC Gendle, M Richardson, J Leeper, LB Hardin, JM Green, and PA Bishop. Wheelchair-mounted accelerometers for measurement of physical activity. Disability and rehabilitation. Assistive technology, 7(2):139, 2012.

[7] S. V. Hiremath and D. Ding. Regression equations for RT3 activity monitors to estimate energy expenditure in manual wheelchair users. In 2011 Annual International Conference of the IEEE Engineering in Medicine and Biology Society, pages 7348-7351, Aug 2011. 
[8] Shivayogi Vishwanath Hiremath and Dan Ding. Evaluation of activity monitors in manual wheelchair users with paraplegia. The journal of spinal cord medicine, 34(1):110-117, 2011.

[9] Peter Kokkinos, Helen Sheriff, and Raya Kheirbek. Physical inactivity and mortality risk. Cardiology Research and Practice, 2011.

[10] Tom Edward Nightingale, Jean-Philippe Walhim, Dylan Thompson, and James L. J. Bilzon. Predicting physical activity energy expenditure in manual wheelchair users. Medicine and Science in Sports and Exercise, 46(9):18491858, September 2014.

[11] Amit Pande, Yunze Zeng, Aveek Das, Prasant Mohapatra, Sheridan Miyamoto, Edmund Seto, Erik K. Henricson, and Jay J. Han. Accurate energy expenditure estimation using smartphone sensors. In Proceedings of the 4th Conference on Wireless Health, WH '13, pages 19:1-19:2, New York, NY, USA, 2013. ACM.

[12] Russell R Pate, Michael Pratt, Steven N Blair, William L Haskell, Caroline A Macera, Claude Bouchard, David Buchner, Walter Ettinger, Gregory W Heath, Abby C King, et al. Physical activity and public health: a recommendation from the centers for disease control and prevention and the american college of sports medicine. Jama, 273(5):402-407, 1995.

[13] F. Pedregosa, G. Varoquaux, A. Gramfort, V. Michel, B. Thirion, O. Grisel, M. Blondel, P. Prettenhofer, R. Weiss, V. Dubourg, J. Vanderplas, A. Passos, D. Cournapeau, M. Brucher, M. Perrot, and E. Duchesnay. Scikitlearn: Machine learning in Python. Journal of Machine
Learning Research, 12:2825-2830, 2011.

[14] John Rooksby, Mattias Rost, Alistair Morrison, and Matthew Chalmers Chalmers. Personal tracking as lived informatics. In Proceedings of the 32Nd Annual ACM Conference on Human Factors in Computing Systems, CHI '14, pages 1163-1172, New York, NY, USA, 2014. ACM.

[15] Nicole Ruch, Franziska Joss, Gerda Jimmy, Katarina Melzer, Johanna Hänggi, and Urs Mäder. Neural network versus activity-specific prediction equations for energy expenditure estimation in children. Journal of Applied Physiology, 115(9):1229-1236, 2013.

[16] Skipper Seabold and Josef Perktold. Statsmodels: Econometric and statistical modeling with python. In 9th Python in Science Conference, 2010.

[17] John Staudenmayer, David Pober, Scott Crouter, David Bassett, and Patty Freedson. An artificial neural network to estimate physical activity energy expenditure and identify physical activity type from an accelerometer. Journal of applied physiology (Bethesda, Md. : 1985), 107(4):1300-1307, October 2009.

[18] Ricardo A. Tanhoffer, Aldre I. P. Tanhoffer, Jacqueline Raymond, Andrew P. Hills, and Glen M. Davis. Comparison of methods to assess energy expenditure and physical activity in people with spinal cord injury. The Journal of Spinal Cord Medicine, 2012.

[19] Nisarg Vyas, Jonathan Farringdon, David Andre, and John Stivoric. Machine learning and sensor fusion for estimating continuous energy expenditure. AI Magazine, 33(2), 2012. 